\title{
Research on Image Processing Algorithm in High Resolution Infrared Imaging Based on Principal Component Analysis
}

\author{
Fujun Wang \\ Dongchang College of Liaocheng University, Liaocheng, Shandong, 252000, China
}

Keywords: Principal component analysis, Infrared imaging, Image processing, Image interpolation

\begin{abstract}
High resolution imaging has always been the focus of research in the field of infrared imaging. It is not only of great significance in theory, but also has urgent needs in practice. The nonuniformity correction methods commonly used in image processing are analyzed and studied. Through analyzing the advantages and disadvantages of calibration-based and scene-based correction methods, a nonuniformity correction method of two points+high pass in time domain is proposed. The mechanism of non-uniformity is discussed from three aspects of infrared focal plane array, readout circuit and ambient temperature, and the exponential relationship model between detector response and ambient temperature is established. Aiming at the problem of detector nonuniformity drifting with time, three improved scene nonuniformity correction algorithms are proposed. Aiming at the contradiction between large dynamic range of signal source and small dynamic range of display output in infrared focal plane array signal processing, an adaptive histogram equalization technology for infrared images is proposed. The limitation of the dynamic range on the performance of the infrared focal plane array system is solved, and the image quality is improved.
\end{abstract}

\section{Introduction}

Infrared imaging technology is one of the high technologies developed vigorously in the world today and is widely used in military and civil fields. Limited by manufacturing process, materials and other factors, the response of infrared focal plane array detection unit is inconsistent, leading to principal component and non-uniform noise, which reduces the temperature resolution of the system [1]. Infrared and visible light are the two forms of electromagnetic radiation. Their natural essence is the same. The difference between them is only the wavelength and frequency. For an object with a temperature above absolute zero, its own molecules and atoms will move irregularly, thus continuously radiating infrared heat energy to the outside, and the magnitude of this heat radiation energy is directly related to the temperature of the surface of the object. Compared with radar systems, infrared imaging systems are small in size, light in weight and low in power consumption, and are especially suitable for precision guided weapons [3]. Under normal circumstances, the contrast of the image is relatively low, the details of the target are difficult to distinguish, and the feature information contained in the image is relatively fuzzy. These features are very obvious in civil detection and monitoring system application fields. The useless information should be weakened or removed, while the low-resolution infrared image should be enlarged, the clarity should be ensured as much as possible, and an appropriate segmentation method should be adopted when extracting the target from complex background and noise interference. Therefore, it is necessary to study the infrared image preprocessing algorithm.

\section{Pre-Filtering of Micro-Scan Images}

\subsection{Isotropic Diffusion Equation}

Filtering the image to reduce the influence of noise is an important link in image processing. In general, neighborhood averaging is used in the spatial domain to reduce noise in the frequency domain. Since the noise spectrum is distributed in the high frequency band, low-pass filtering is 
used to reduce noise [4]. The infrared imaging system without exception has the problems of poor image uniformity, large number of principal components, unstable response and low image contrast. Its solution is given in the form of a system function or impulse response obtained under the condition of minimum mean square error, while Kalman filter only needs to estimate the current value of the signal by using the estimation of the previous state and the measurement data of the current state, and does not need all the past measurement data. Based on anisotropic diffusion theory, an improved noise suppression algorithm is discussed. Using derivative operator to extract edge point set of noise image.

Assume the first. The input image $I(x, y, 0)$ at time, then the output image $I(x, y, t)$ at time $\mathrm{t}$ is expressed as follows: [5]

$$
\begin{array}{r}
\frac{\partial I(x, y, t)}{\partial t}=I(x, y, 0) * G(x, y, t) \\
G(x, y, t)=\frac{1}{2 \pi t^{2}} e^{-\frac{x^{2}+y^{2}}{2 t^{2}}}
\end{array}
$$

Among them, $G(x, y, t)$ is a Gaussian operator, and t is a scale space. Koenderink and Hummel proved that the above formula is equivalent to the solution of the heat conduction (isotropic diffusion) equation. The expression of the isotropic diffusion equation is as follows:

$$
\frac{\partial I(x, y, t)}{\partial t}=\Delta I(x, y, t)=c \cdot\left(\frac{\partial^{2} I(x, y, t)}{\partial x^{2}}+\frac{\partial^{2} I(x, y, t)}{\partial y^{2}}\right)
$$

Among them, * is a convolution operation, $G(x, y, t)$ is a Gaussian convolution kernel, and c is a diffusion coefficient.

Calibration based on reference source is to provide uniform infrared radiation by setting reference source and calculate corresponding calibration parameters by measuring response through calibration method. The implementation of the algorithm is simple, the degree of engineering of the algorithm is high, and good correction effect can be obtained after correction. However, band noise is mainly caused by nonuniformity of response rate of infrared detector itself, imaging defects and clutter interference. Various sources and types of noise cause the complexity of infrared thermal image noise [6].

\subsection{Anisotropic Diffusion Equation}

Isotropic diffusion filtering will lose the details of the image target while smoothing the noise. The basic idea of Perona and Malik anisotropic diffusion algorithm is to define the original image I0 on the convex domain $\Omega \subset R \times R$. The non-uniformity of the original collected image of infrared imaging system is generally $1 \% \sim 15 \%$, and the non-uniformity of the system image is generally less than $0.8 \%$ under the environmental conditions. The non-uniformity of the image severely restricts the overall performance of the system [7]. During the period of obtaining an observation vector called vector time, the gain and bias of the detector are approximately constant, while during the subsequent time of obtaining other observation vectors, these parameters may have drifted.

Diffusion occurs at a variable rate, thus obtaining a series of smooth images $I(x, y, t)$. the anisotropic diffusion equation expression is as follows: [8]

$$
\left\{\begin{array}{c}
\frac{\partial I(x, y, t)}{\partial t}=\operatorname{div}[g(|\Delta I|) \cdot \Delta I(x, y, t)] \\
\left.I(x, y, t)\right|_{t=0}=I(x, y, 0)
\end{array}\right.
$$

Where $I(x, y, t)$ is the output image, $\Delta$ is the gradient operator, div is the divergence operator, and $g($.$) represents the diffusion coefficient (non-bounded decreasing function) and is a function of$ 
the gradient amplitude $|\Delta I|$ of the local image. Perona and Malik give two diffusion functions expressed as follows:

$$
\begin{aligned}
& g(s)=\exp \left[-(s / k)^{2}\right](5) \\
& g(s)=1 /\left(1+(s / k)^{2}\right)(6)
\end{aligned}
$$

Among them, the constant $\mathrm{k}$ is appropriately adjusted according to different application scenarios.

The response of infrared detector is linear and stable. The response of infrared detector is linear in most dynamic range, but it is not linear only near the cut-off and saturation regions of the detector response. Ignoring the limit conditions at both ends of the probe response, it can be considered as linear, and the probe response remains stable within a certain period of time. In actual infrared detection, the detection target is often not stationary, and there is a lot of moving target information. The simple inter-frame filtering algorithm introduced above only has good noise reduction effect for still infrared images, and has certain defects for moving infrared targets.

\section{Image Interpolation Based on Edge Preservation}

\subsection{Local Standard Deviation Model}

Edge and texture features are important information of images, which are represented as detail components. In the process of image interpolation, protecting edge features is an important link [9]. According to the spatial neighborhood and pixel correlation characteristics of pixel neighborhood, an improved edge protection image interpolation algorithm is proposed. The output of the detection unit is quite different and needs non-uniform correction. According to the response characteristics of the infrared detector, each pixel has different gain coefficient and offset coefficient. The pixel needs non-uniform correction through the corresponding gain coefficient and offset coefficient. That is, the gain and offset of the detector in the infrared focal plane array are Gaussian random variables and change slowly and randomly between continuous observation vectors, so GaussianMarkov process is used to establish the state equation model.

The standard deviation reflects the contrast of the local area, assuming that the image size is $\mathrm{N} \times \mathrm{M}, f_{i, j}$ is the pixel of position (i,j), and $\mathrm{w}$ is the window of $\mathrm{d} \times \mathrm{d}$ centered on (i, j). Then, when the

window $\mathrm{W}$ slides in the image, the standard deviation ${ }^{i, j}$ of the center (i,j) of its overlapping region is shown by the following formula:

$$
\begin{array}{r}
v_{i, j}=\sqrt[1]{\frac{1}{d} \sum_{p=i-\frac{d-1}{2} q=j-\frac{d-1}{2}}^{i+\frac{d+1}{2}}\left(f_{p, q}-\bar{m}_{i, j}\right)^{2}} \\
\bar{m}_{i, j}=\sum^{\frac{d+1}{2}} \sum^{i+\frac{d+1}{2}} \sum^{j+\frac{d+1}{2}} f_{p, q} \\
2
\end{array}
$$

Where $f_{p, q}$ represents the pixel value of the sliding window position (p,q), ${ }^{\bar{m}_{i, j}}$ represents the sliding window pixel mean, $0 \leq i, p \leq N-1,0 \leq j, q \leq M-1$.

It is assumed that the excitation noise of the gain and the excitation noise of the bias are Gaussian distribution, and the two are independent and uncorrelated. The size of an object in an infrared image is often larger than 1 pixel, and the movement of the object is often manifested in the 
overall change of pixel gray levels in the neighborhood of $n^{*} n$ around the object, so the judgment of a single pixel has limitations. The gray scale transformation function is thus obtained. Finally, the function is applied to enhance the central pixel point in the neighborhood. Next, the center of the neighborhood is transferred to the adjacent pixels, and the previous steps are repeated.

\subsection{Edge-Preserving Image Interpolation}

The main idea of the interpolation model based on edge preservation is firstly to judge whether the current pixel belongs to a smooth area or an edge area, and secondly to interpolate for the smooth area and the edge area respectively. Image acquisition is carried out by setting the high and low temperature of the blackbody, correction coefficients calculated according to the above formula are stored in the product, and when the system works, the stored correction coefficients are read to carry out nonuniformity correction processing on the image.

If the standard deviation of the neighborhood of the current pixel point is greater than the set threshold, it indicates that the current pixel point belongs to an edge point. At this time, the pixel point in the maximum gradient direction is selected to replace the current pixel point. The interpolation model is as follows

$$
\begin{gathered}
\left\{\begin{array}{c}
f_{i, j}=a_{i, j-1} f_{i, j-1}+a_{i, j+1} f_{i, j+1}+a_{i-1, j} f_{i-1, j}+a_{i+1, j} f_{i+1, j},<g_{i, j}<T> \\
f_{i, j}=f_{M},<g_{i, j}>T>
\end{array}\right. \\
M=\max \left(\left|f_{i, j-1}-f_{i, j}\right|,\left|f_{i, j+1}-f_{i, j}\right|,\left|f_{i-1, j}-f_{i, j}\right|,\left|f_{i+1, j}-f_{i, j}\right|\right)
\end{gathered}
$$

Among them, $\mathrm{T}$ is the threshold, $\mathrm{M}$ is the modulus of the maximum direction derivative, ${ }^{a_{i, j+1}}$, $a_{i, j-1}, a_{i+1, j}$ and $a_{i-1, j}$ are the weighting coefficients of each direction, $f_{i, j}$ is the pixel value of position (i, j), $\left|f_{i-1, j}-f_{i, j}\right|$ is the pixel position (i, j ) and (i-1, j).

The flow chart of the infrared image edge enhancement algorithm based on multi-scale morphological wavelet transform is shown in Fig. 1. using each high-frequency subband image after nonlinear enhancement, image reconstruction is carried out by using equations (9) and (10), and finally an infrared image with enhanced target edge is obtained.

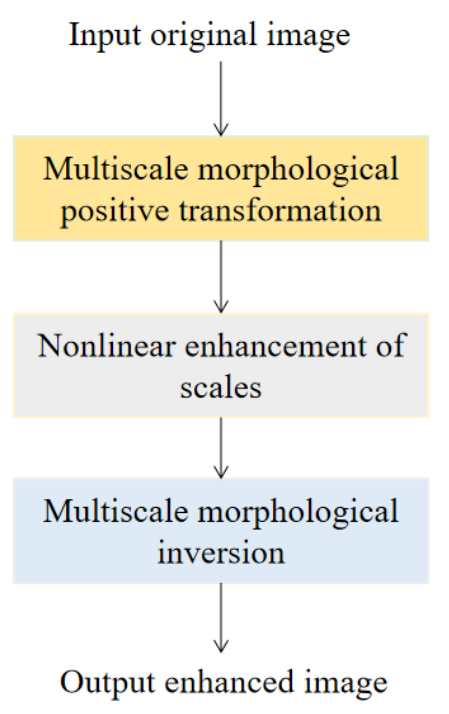

Fig.1 Algorithm Flow

In the actual imaging system work, the observation vector of each detection unit in the infrared focal plane array, that is, the "frame block" mentioned, can be obtained by measurement, so the vectorized linear measurement equation model is adopted in the algorithm in this chapter. The larger the value is, the greater the degree of target motion is. Therefore, the weight of the pixel gray 
level of the current frame in the output image should be increased instead of a simple average when performing inter-frame filtering. It will have a negative effect on the processing results. In application, the window size should be selected according to the requirements. The selection of window size is based on visual effects. Large windows have better effects, while small windows make the local details of the image more prominent. The description of the imaging process is more accurate, and the solution accuracy and anti-noise capability are better than those of the spatial domain model, which is helpful for the more accurate description and solution of the problem. This is the significance of frequency domain method research.

\section{Super-Resolution Reconstruction of Multi-Frame Images}

\subsection{Frequency Domain Reconstruction}

Based on the frequency domain de-aliasing theory, the super-resolution reconstruction of images is carried out by using the translation and aliasing characteristics of Fourier transform of continuous and discrete signals. A set of equations consisting of translation and confusion parameters between low-resolution images and Fourier coefficients of low-resolution and high-resolution images is solved. The response characteristics of infrared imaging change slowly with time and pixel 1/f noise are all of low frequency, while the target has a certain moving speed in the background, so the target belongs to high frequency. In addition, in practice, if a set of measurement frame blocks obtained by receiving infrared radiation input of approximately the same range are used to estimate the state vector, the comparison of images after adding noise and images after removing noise shows that the algorithm in this paper can effectively suppress various noises existing in the images. When a small window is used for image enhancement, the degree cannot be well controlled. Although details are highlighted, more noise will inevitably be added. There are both advantages and disadvantages. Compared with small windows, there is no absolute difference between good and bad. High resolution images can be obtained by direct reconstruction using them to improve the spatial resolution of the system. This global method is applicable to the enhancement of the whole image, but sometimes it is also necessary to locally enhance the details of small areas of the image so as to maintain the integrity and clarity of the content.

\subsection{Airspace Reconstruction}

Spatial reconstruction algorithm is flexible and has the ability to contain spatial prior constraints, including nonuniform spatial sample interpolation, iterative back projection (IBP), convex set projection (POCS), etc. However, it is required that the response of the infrared imaging system has a good linearity, and that the target maintains a high relative motion speed, and that the convergence parameter $\mathrm{M}$ should be selected by comprehensively considering two factors of not weakening the target and correcting the convergence speed.

Assuming that $\mathrm{f}(\mathrm{x}, \mathrm{y})$ represents a high-resolution image, $\mathrm{F}(\mathrm{u}, \mathrm{v})$ is a continuous Fourier transform of $f(x, y)$, the positional relationship between the k-th image $f k(x, y)$ and $f(x, y)$ is as follows:

$$
f_{k}(x, y)=f\left(x+\Delta_{x k}, y+\Delta_{y k}\right)
$$

Assuming that the sampling periods of the image $f_{k}(x, y)$ in the horizontal direction and the vertical direction are $T_{1}$ and $T_{2}$, respectively, the low-resolution image expression is as follows:

$$
g_{k}(x, y)=f_{k}\left(m T_{1}, n T_{2}\right)
$$

Then the frequency domain relation expression of the high-resolution image $f_{k}(x, y)$ and the kth low-resolution image is as follows: 


$$
G_{k}(u, v)=\frac{1}{T_{1} T_{2}} \sum_{m} \sum_{n} F_{k}\left[\frac{2 \pi}{T_{1}}\left[\frac{u}{M^{\prime}}+m\right], \frac{2 \pi}{T_{2}}\left[\frac{v}{N^{\prime}}+n\right]\right]
$$

The above formula is abbreviated as

$G=\Phi F(14)$

Where g represents the Fourier transform coefficient of the low-resolution image, $\mathrm{f}$ represents the Fourier coefficient of the high-resolution image, and the coefficient matrix $\Phi$ represents the amount of phase change between different low-resolution images.

The flowchart of the infrared image edge enhancement and segmentation algorithm based on lifting format wavelet transform and Snake model is shown in Fig. 2, and the implementation steps are summarized as follows [10]

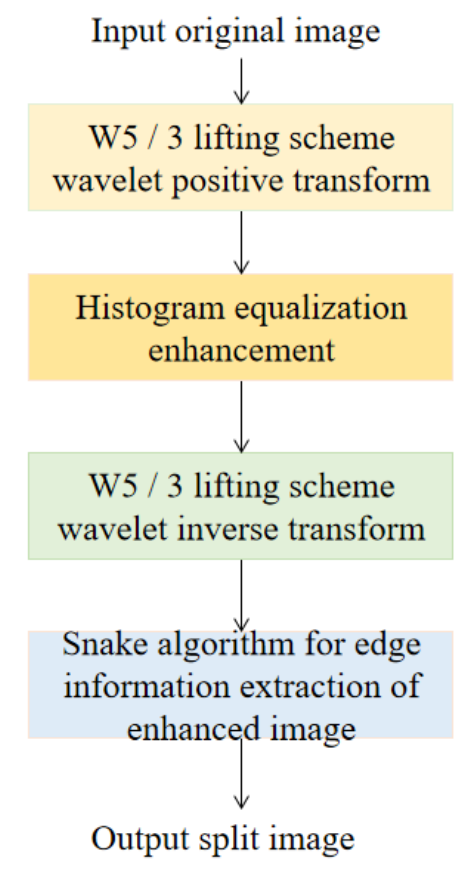

Fig.2 Algorithm Flow

In this case, if the above-mentioned linear response model is still adopted, the nonlinearity of the response of the detection unit will have a greater impact on the nonuniformity correction, resulting in a greater error in the correction and a greater residual nonuniformity, thus significantly reducing the imaging quality. During the operation of the infrared focal plane array, new principal components will also appear. At the same time, the image data will be interfered in the transmission process to generate interference points. The spatial distribution of these principal components and interference points is more similar to salt and pepper noise. There is an obvious difference with the global histogram equalization. Many details in this region are lost, which is caused by the statistical information in other regions. That is to say, the corresponding gray scale range has changed, but all the details included in the local histogram equalization have been strengthened.

\section{Conclusion}

Based on principal component analysis, this thesis aims to improve the temperature resolution and spatial resolution of the infrared focal plane array and the imaging quality of the infrared system, mainly focusing on nonuniformity correction and super-resolution reconstruction of infrared imaging. On the basis of fully studying the existing image processing algorithms, two-point and time-domain high-pass nonuniformity correction algorithms, principal component detection algorithms based on median filtering and histogram statistics, infrared image enhancement algorithms based on gray scale stretching algorithms, and image enhancement high-pass filtering algorithms are studied and proposed. The algorithm uses Gaussian-Markov process to model the 
gain and bias of each detector in the infrared focal plane array, establishes the state equation and measurement equation, thus obtains the iteration formula of nonuniformity correction based on Kalman filter, and realizes nonuniformity correction of images. The edge region uses the pixel point with the maximum gradient direction to realize image interpolation. Polynomial interpolation reconstruction divides the image into a plurality of same-sex regions according to local gradients, and adaptively adjusts the order in the same-sex regions to complete image interpolation reconstruction.

\section{Acknowledgement}

The authors acknowledge the Shandong University Science and technology plan project (Grant: J17KB145).

\section{References}

[1] Zhang Liang. (2017). A fusion method of battlefield infrared and visible gray reconnaissance image. Naval electronic engineering, vol. 37, no. 12, pp. 76-80.

[2] Wang Pengfei. (2017). Influencing factors and enhancement methods of infrared image. Rural science experiment, no. 3, pp. 88-89.

[3] Ran Yunchao, appeal, Zhou Bin, et al. (2018). Research on the modeling method of infrared detector imaging realism. Computer and digital engineering, vol. 46, no. 5, pp. 1003-1006.

[4] Shi Hongyun, Xu Lianggang, Guo Tao, et al. (2019). Insulator infrared detection and image processing technology. China Science and technology information, no. 9, pp. 98-99.

[5] Wang Xiaofang, Kang Chen, Cheng Hongbo, et al. (2019). Automatic diagnosis method of thermal fault of substation equipment based on infrared image processing. Journal of East China Jiaotong University, no. 3, pp. 111-118.

[6] Li Shengjun, Zheng Zhu. (2017). Detection algorithm of light leakage defects on workpiece surface based on micro imaging and image processing. Modular machine tool and automatic processing technology, no. 6, pp. 141-144.

[7] Jia Mingfeng, Hu Guoqing, LV Chengzhi. (2017). Research on automatic glue spraying system based on image processing. Manufacturing automation, vol. 39, no. 6, pp. 116-119.

[8] Luo Bin. (2019). Two dimensional deformation method for interpolation of fault image. Chinese Journal of image graphics, vol. 3, no. 8, pp. 675-678.

[9] Wang Xiao, Guan Zhiqiang, Wang Jing, et al. (2019). Color image sonar target detection based on convolutional neural network. Computer application, no. A01, pp. 187-191.

[10] Li Baoshan, Dai Renhui, Liu Ke, et al. (2019). Application of MATLAB in audio, image noise and data fitting. Computer and information technology, no. 3, pp. 10-14. 